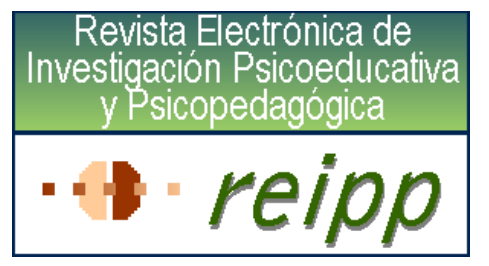

REVISTA ELECTRÓNICA DE INVESTIGACIÓN PSICOEDUCATIVA Y PSICOPEDAGÓGICA

No 1 (2) 2003. ISSN: 1696-2095

\title{
Contexto social, género y competencia social autoevaluada en alumnos andaluces de 11-12 años
}

\author{
María Victoria Trianes Torres* \\ María Cardelle-Elawar** \\ María José Blanca Mena* \\ Ángela M. Muñoz Sánchez* \\ * Universidad de Málaga / ** Arizona State University West \\ España / EE.UU.
}

triatorr@uma.es 


\section{RESUMEN}

Introducción. Aunque el área de estudio de la Competencia social está hoy plenamente consolidada, aun son precisos estudios que dibujen las circunstancias y matices que median en los resultados de las distintas edades. Particularmente, la autoevaluación de competencia social representa un punto de vista necesario y que ha sido menos estudiado que la evaluación realizada por el profesor o los iguales, refiriéndonos al contexto escolar. El objetivo de este trabajo ha sido examinar las diferencias en diversas medidas de competencia social en estudiantes de $6^{\circ}$ de primaria (11-12 años) que asisten a tres escuelas pertenecientes a diferentes contextos socioculturales: deprimido, medio y residencial. Se han examinado también las diferencias por género.

Método. Administramos una serie de cuestionarios que medían las habilidades sociales apropiadas e inapropiadas, empatía, agresividad, asertividad y pasividad hacia adultos y hacia iguales. Género y contexto social han sido las variables independientes consideradas para los análisis. Las variables dependientes han sido 12 habilidades sociales y problemas conductuales que forman la medida compuesta de competencia social utilizada.

Resultados. Los resultados muestran que la empatía, asertividad inapropiada y celos/aislamiento varían en función de la interacción de género y el contexto social. El único efecto principal debido al género se ha encontrado en la variable impulsividad, indicando puntuaciones más altas en los chicos. El efecto principal del contexto social ha resultado más potente que el del género, revelando también diferencias en diversas variables no implicadas en la interacción.

Discusión. Las diferencias relativas al contexto social son interpretadas en función de variaciones culturales relativas a los criterios con los que se juzga la conducta social hábil, debido a características diferenciales entre contextos deprimidos y acomodados, que se reflejan en diferentes niveles de exigencia respecto al logro de objetivos sociales asociados a la educación de niños y adolescentes, en casa y en los contextos escolares.

Palabras Clave: Competencia Social autoinformada, preadolescentes, género, contexto social. 


\section{Introducción}

La competencia social es un constructo complejo. Su definición implica diversos puntos de vista que operacionalizan aspectos diversos. A pesar de sus diferencias, la mayoría de los estudios están de acuerdo en que implica un funcionamiento efectivo en los contextos sociales (Cavell, 1990). Competencia Social es, pues, un amplio y relevante campo de desarrollo, que tiene un importante peso en la adaptación y ajuste psicológico (Cichetti y Bukowiski, 1995; Parker, Rubin, Price y DeRosier, 1995), como campo o tarea del desarrollo (i.e. Asher y Parker, 1989, 1991) así como es objetivo educativo de primera magnitud (en nuestro país, Monjas, 2002; Trianes, 1996; Trianes, Blanca, Muñoz, García, Cardelle-Elawar e Infante, 2002; Trianes y Fernández-Figarés, 2000; Trianes y García Correa, 2002).

Desde la evaluación psicoeducativa, una de las definiciones más extendidas considera la competencia social como la suma de los juicios emitidos sobre un niño por los agentes sociales relevantes. Lejos de considerarla un constructo estrictamente asociado a habilidades sociales específicas, predomina hoy la idea de que la competencia social puede considerarse una competencia de carácter más general (McConnell y Odom, 1986; Cavell, 1990), incluso puede ser entendida como un factor general de carácter transituacional (Bracken y Crain, 1994).

El papel del contexto social es muy importante en la evaluación de la conducta social. Ésta se ajusta a los criterios del contexto, ya que los evaluadores valoran con arreglo a ellos y las personas, a todas las edades, conocen estos criterios de referencia y saben que van a ser evaluados con ellos. Por ello, la evaluación de la conducta hábil recoge influencias del género, la edad, el papel o rol del que habla, las situaciones y contextos específicos en los que se desarrolla la conducta, y otras variables que diferencian comportamientos hábiles de los inhábiles, siempre según los contextos.

Por tanto la medida de la competencia social se aloja en contextos específicos culturales y comunitarios. En la investigación existe consenso en la creencia de que diferentes contextos se asocian a diferentes resultados en la competencia social infantil. El contexto impacta sobre los factores de riesgo de dos maneras (Soriano y Soriano, 1994). Primero, 
puede proteger frente a factores de riesgo proporcionando normas, valores, un sistema de apoyo, ayudando a afirmar un sentido positivo del Yo y proporcionando un modelo de vida. En segundo lugar, puede fallar en esta protección frente al riesgo cuando queda debilitado por factores hostiles sociales, económicos, o por procesos de exclusión de la sociedad dominante cayendo en culturas marginales (Roberts, Garritz y Kearney, 1990).

Existe numerosa bibliografía que pone en relación la existencia de comportamientos agresivos y violentos en adolescentes y jóvenes, con entornos de pobreza, estatus socioeconómico bajo con presencia de problemas económicos y en entornos con escasos recursos comunitarios (Guerra, Huesmann, Tolin, Acker y Eron, 1995). También se ha encontrado efectos asociados a interacciones entre género y status socioeconómico. Según estas diferencias los chicos parecen mostrar más problemas sociales que las chicas en familias de bajo status socioeconómico (Jaffe, Wolfe, Wilson, y Zak; 1985; Verhalst, Akkerhuis y Althaus, 1985; Heimer, 1997).

Específicamente, la autoevaluación muestra características peculiares que la diferencian de la evaluación que hacen agentes externos (profesores e iguales) (Trianes, Blanca, Muñoz, García, Cardelle-Elawar e Infante, 2002). Alumnos y profesores pueden evidenciar distintos criterios para juzgar la conducta competente (Trianes, Muñoz, Blanca, Sánchez, y García, 1999). Alumnos adolescentes con conducta agresiva pueden mostrar sesgos al autoevaluar su competencia social (Baumeister, Smart y Boden, 1996; Colvin, Bloch y Funder, 1996; Raskin, Novacek y Hogan, 1991; Salmivalli, Kaukiainen, Kaistaniemi y Lagerspetz, 1999). También el género es fuente de diferencias en la autoevaluación de habilidades sociales estando conectados género y autoconcepto de diversas maneras (Koestner y Aube, 1995).

El presente estudio trata de analizar las diferencias producidas en función del género, el contexto social y la interacción de estos dos factores, en aspectos específicos de la competencia social autoevaluada. Estos aspectos son: habilidades sociales apropiadas, asertividad, agresividad y pasividad hacia los adultos y hacia los iguales, empatía, asertividad inapropiada, impulsividad, celos/ soledad y sobreconfianza. Es también común que la investigación sobre efectos del género y contexto social sobre la competencia social incluya habilidades sociales y conductas antisociales como medidas de referencia (Prior, Smart, 
Sansón y Oberklaid, 1993; Soriano and Soriano, 1994). Por esa razón, en este trabajo analizamos la competencia social a partir de medidas autoevaluadas de alumnos de 11-12 años que incluyen habilidades y problemas de la conducta social.

\section{Método}

\section{Participantes}

Los participantes han sido 96 alumnos (de 11-12 años) de $6^{\circ}$ grado procedentes de tres contextos diferentes: deprimido, medio y residencial. 50 eran niños y 46 eran niñas. Las tres escuelas y sus profesores correspondientes fueron voluntarias para participar en este estudio. Todas ellas eran de Málaga capital (España). La primera escuela, en un área deprimida de la ciudad incluye 21 alumnos ( 7 niños y 14 niñas) de familias con algún tipo de problemas y bajo estatus socio-económico; la segunda escuela incluye 45 alumnos distribuidos en dos clases (25 niños y 20 niñas). Es una escuela privada concertada en un área de status socioeconómico alto; y la tercera escuela incluye 27 alumnos (18 niños y 9 niñas ) de un nivel socio-económico medio. Aunque no hemos evaluado con detalle el contexto social, estas tres escuelas están en tres barriadas que diferencian su contexto por numerosas indicadores sociales, económicos y culturales, que son bien conocidos en la ciudad.

\section{Procedimiento}

Género y contexto social han sido las variables independientes consideradas para los análisis. Las variables dependientes han sido 12 habilidades sociales y problemas conductuales que forman la medida compuesta de competencia social utilizada. Los instrumentos fueron aplicados a los participantes a mitad del curso. Se seleccionó este momento asumiendo que los alumnos podrían estar mejor adaptados a la escuela, sin las tensiones típicas de los comienzos del curso. Todos los tests fueron presentados a todos los alumnos en sus clases, en dos sesiones en días diferentes. Tuvieron un descanso de una hora en cada sesión.

\section{Instrumentos}

Los siguientes instrumentos utilizados evalúan las variables desde el punto de vista del propio participante. 


\section{1) Cuestionario Matson Evaluation of Social Skills with Youngsters} (castellano)(MESSY), desarrollado por Matson, Rotatori and Helsel (1983), mide la extensión en la que un niño o niña emplea habilidades sociales apropiadas durante su interacción en clase. Es un conocido instrumento en el área de la competencia social. Sus propiedades psicométricas han sido investigadas profusamente, particularmente en muestras angloparlantes, resultando, en general, satisfactorias (Helsen y Matson, 1984; Kazdin, Matson y Elveldt- Dawson, 1984; Matson, Macklin y Helsel, 1985; Matson y Ollendick, 1988; Spence y Liddle, 1990), y en muestras españolas (Mendez, Hidalgo e Ingles, 2002). Para su utilización, en este estudio, se tradujeron y adaptaron los items de esta prueba en el seno del propio equipo de trabajo. La consistencia interna evaluada en este estudio por medio del alpha de Cronbach ha sido de .89

El cuestionario dirigido a alumnos presenta un formato de respuesta en cuatro escalones: nunca, a veces, a menudo y siempre. Se han considerado los cinco factores siguientes:

- Habilidades Sociales Apropiadas (24 ítems). Este factor evalúa conductas como expresividad emocional, tener amigos, compartir, etc. Algunos ejemplos de items son: "miro a la gente cuando hablo con ella" y "me dirijo a la gente y entablo conversación."

- Asertividad Inapropiada (16 items), el cual explora conductas agresivas, de burla o abuso de los demás. Algunos ejemplos de items son: "cojo cosas que no son mías sin permiso" y "pego cuando estoy furioso."

- Impulsividad (5 items). Algunos ejemplos de items son: "me enfado fácilmente" e “interrumpo a los demás cuando están hablando.”

- Sobreconfianza (6 items). Este factor explora conductas de sobrevaloración del Yo. Algunos ejemplos de items son: "me gusta presumir ante los demás de las cosas que tengo" y "creo que lo sé todo."

- Celos/Soledad (4 items), el cual explora conductas como sentirse solo o no tener amigos.

2) EI Índice de Empatía para niños y adolescentes desarrollado por Bryant, (1982) tiene 22 items que se responden con un formato de SI/NO. Por ejemplo: "Me siento triste si oigo llorar a otro niño." El autor evalúa su consistencia interna por medio del Alpha de Cronbach obteniendo un .68 para clases de séptimo grado. La fiabilidad Test-retest fue 
$\mathrm{r}(108)=-81$ para cuarto grado utilizando el formato SI/NO y $\mathrm{r}(80)=.83$ usando un formato en nueve escalones de respuesta en el índice de empatía para adolescentes.

3) La Children's Assertiveness Behaviour Scale (CABS) desarrollada por Michelson, Wood, and Kazdin (1983), mide tres tipos de conductas: asertiva, agresiva y pasiva. Las situaciones, seis en total se refieren tanto a la interacción con adultos como con iguales. En esta medida autoinformada los alumnos seleccionan la conducta que podrían presentar en una hipotética situación problemática. El formato supone que, en cada problema, el niño debe elegir entre cinco alternativas de respuesta, dos son agresivas, dos pasivas y una asertiva. Las seis situaciones que se ofrecen al participante son: 1) expresar y responder a manifestaciones positivas; 2) expresar y responder a manifestaciones negativas; 3 ) dar y seguir órdenes y peticiones; 4) iniciar y mantener conversación y escuchar a los demás durante la conversación; 5) expresar y reaccionar ante los sentimientos y manifestaciones de comprensión. Las seis puntuaciones que arroja la prueba son:

- Asertividad con iguales

- Asertividad con adultos

- Pasividad con iguales

- Pasividad con adultos

- Agresividad con iguales

- Agresividad con adultos

\section{Resultados}

Se ha realizado un análisis multivariante de la varianza $2 \times 3$ con las variables provenientes del MESSY, CABS y de la escala de empatía. Los factores fueron el género y el contexto social, con tres niveles: deprimido, medio y residencial. Los resultados mostraron significación estadística, siguiendo la Lambda de Wilks, para el efecto principal del género $[\mathrm{F}(24,150)=10.11 ; \mathrm{p}<.01]$, contexto social $[\mathrm{F}(12,75)=4.3 ; \mathrm{p}<.01]$, así como la interacción de ambos factores $[\mathrm{F}(24,150)=1.9 ; \mathrm{p}=.01]$.

Para averiguar la contribución de cada variable dependiente a la interacción y a los efectos principales, se ha realizado un análisis step-down. Los efectos principales sólo fueron analizados para aquellas variables que no resultaron significativas en la interacción. 
En el análisis step-down para la interacción, se adjudicó prioridad a la variable Asertividad Inapropiada, la cual fue sometida a un análisis univariante de la varianza (ANOVA). Los datos indicaron significación estadística $[\mathrm{F}(2,86)=6.13 ; \mathrm{MSE}=154,24 ; \mathrm{p}<.01]$, mostrando que los chicos tienen puntuaciones mayores que las chicas en el grupo deprimido (figura 1). El resto de variables dependientes se analizaron mediante un análisis de covarianza (ANCOVA), introduciendo como covariables las variables de mayor prioridad (Tabachnik y Fidell, 1989). Así, se realizó un ANCOVA para la variable Empatía con Asertividad Inapropiada como covariable. El análisis reveló una contribución significativa de la misma $[\mathrm{F}(2,85)=3.37 ; \mathrm{MSE}=5.8 ; \mathrm{p}=.039]$. Las medias ajustadas se muestran en la figura 2. Los resultados indican que los chicos tienen menor puntuación en Empatía que las chicas en los grupos deprimido y residencial.

Una vez mostrada las aportaciones de Asertividad inapropiada y Empatía, se realizaron una serie de ANCOVAs con el resto de variables como dependientes y éstas dos como covariables. El único efecto significativo fue para Celos/Soledad $[\mathrm{F}(2,84)=3,24 ; \mathrm{MSE}=7$; $p=.04]$, cuyas medias se muestran en la figura 3. Los datos indican mayores diferencias entre género en el grupo deprimido, donde los niños presentan puntuaciones menores en esta variable.

En el análisis step-down para el género, sólo la variable Impulsividad contribuyó a las diferencias entre chicos y chicas, siendo los primeros $(\mathrm{M}=8.8)$ más impulsivos que las segundas $(\mathrm{M}=7.9)$. Las demás variables no fueron significativas.

En el análisis para el contexto social, la mayor prioridad fue adjudicada a Habilidades Sociales Apropiadas, la cual fue analizada mediante un ANOVA univariado. Los resultados arrojaron significación estadística $[\mathrm{F}(2,89)=30.2 ; \mathrm{MSE}=71,6 ; \mathrm{p}<.01]$, indicando puntuaciones más bajas en el grupo residencial que en lo otros dos. La siguiente variable que contribuyó a las diferencias, con Habilidades Sociales Apropiadas como covariable, fue la Agresividad hacia Adultos $[\mathrm{F}(2,88)=4.8 ; \mathrm{p}=.01]$. Las comparaciones múltiples de las medias ajustadas muestran que el grupo de contexto deprimido posee mayor puntuación que el residencial, no encontrándose diferencias entre éste y el medio. Otras variables significativas fueron: Asertividad hacia Adultos $[\mathrm{F}(2,87)=6.2 ; \mathrm{p}<.01]$, Pasividad hacia Adultos $[\mathrm{F}(2,86)=12.3$; $\mathrm{p}<.01]$, Agresividad hacia Iguales $[\mathrm{F}(2,85)=4.8 ; \mathrm{p}=.01]$ u Sobreconfianza $[\mathrm{F}(2,84)=6.6$; 
$\mathrm{p}<.01]$. Las medias ajustadas se exponen en la tabla 1. Las medias de Asertividad hacia Adultos indican menores puntuaciones en el grupo medio en relación con los otros dos. Respecto a la Pasividad hacia Adultos, el grupo medio muestra mayores puntuaciones que el bajo, y éste a su vez mayores que el grupo residencial. Finalmente, este último grupo presenta mayores puntuaciones en las variables Agresividad hacia Iguales y Sobreconfianza.

Tabla 1. Medias ajustadas de Habilidades Sociales Apropiadas, Agresividad hacia adultos, Asertividad hacia Adultos, Pasividad hacia Adultos, Agresividad hacia Iguales y Sobreconfianza en función del contexto social.

\begin{tabular}{|l|l|l|l|l|}
\cline { 2 - 5 } \multicolumn{2}{|l|}{ Variables } & \multicolumn{2}{l|}{ Contexto social } & $\begin{array}{l}\text { Comparaciones } \\
\text { múltiples }(\mathrm{p}<.05)\end{array}$ \\
\hline Variables & $\begin{array}{l}\text { Deprimido } \\
(1)\end{array}$ & $\begin{array}{l}\text { Medio } \\
(2)\end{array}$ & $\begin{array}{l}\text { Residencial } \\
(3)\end{array}$ & $\begin{array}{l}\text { Comparaciones } \\
\text { múltiples }(\mathrm{p}<.05)\end{array}$ \\
\hline $\begin{array}{l}\text { Habilidades Sociales } \\
\text { Apropiadas }\end{array}$ & 67.89 & 72.86 & 57.41 & $1-3,2-3$ \\
\hline $\begin{array}{l}\text { Agresividad hacia } \\
\text { Adultos }\end{array}$ & 8.63 & 5.62 & 3.83 & $1-3$ \\
\hline $\begin{array}{l}\text { Asertividad hacia } \\
\text { Adultos }\end{array}$ & 13.88 & 10.48 & 14.77 & $1-2,2-3$ \\
\hline $\begin{array}{l}\text { Pasividad hacia } \\
\text { Adultos }\end{array}$ & 13.96 & 17.67 & 10.59 & $\begin{array}{l}1-2,2-3 \\
1-3\end{array}$ \\
\hline $\begin{array}{l}\text { Agresividad hacia } \\
\text { Iguales }\end{array}$ & 6.24 & 6.81 & 12.52 & $1-3,2-3(\mathrm{p}=.06)$ \\
\hline \begin{tabular}{l} 
Sobreconfianza \\
\hline
\end{tabular} & 9.05 & 9.74 & 11.87 & $\begin{array}{l}2-3,1-3 \\
1-2(\mathrm{p}=.06)\end{array}$ \\
\hline
\end{tabular}

Figura 1. Media de Asertividad Inapropiada en función del contexto social y género.

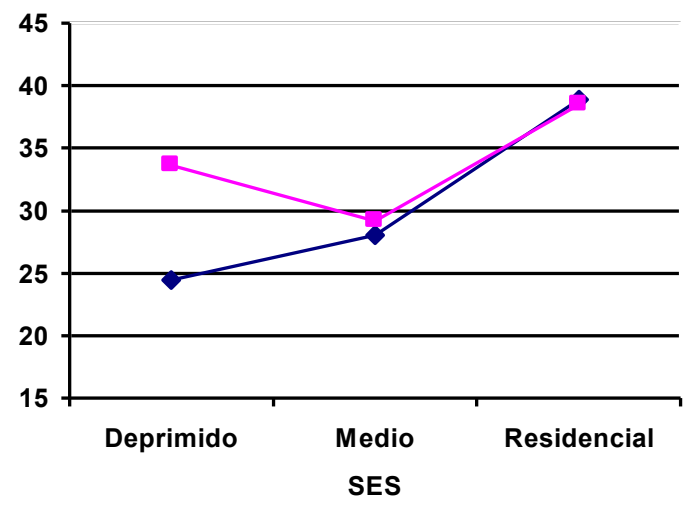

$\longrightarrow$ females $\longrightarrow$ males 
Figura 2. Medias ajustadas en Empatía en función del contexto social y género.

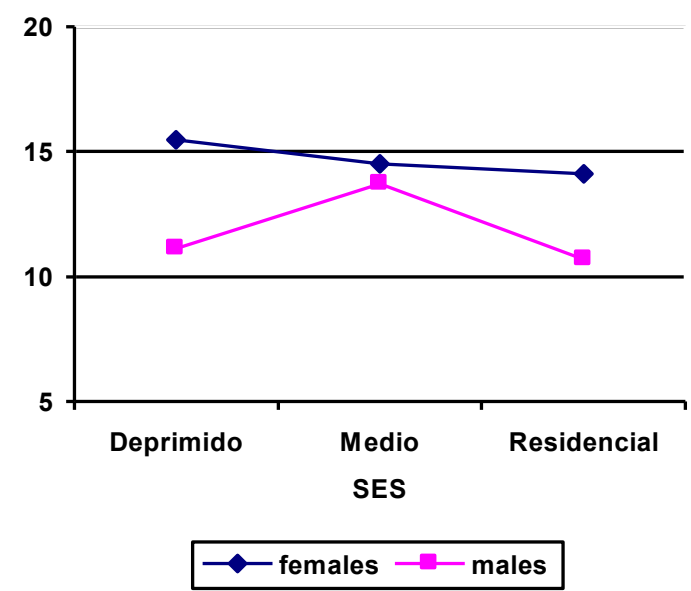

Figura 3. Media ajustada en Celos/ Soledad en función del contexto social y género.

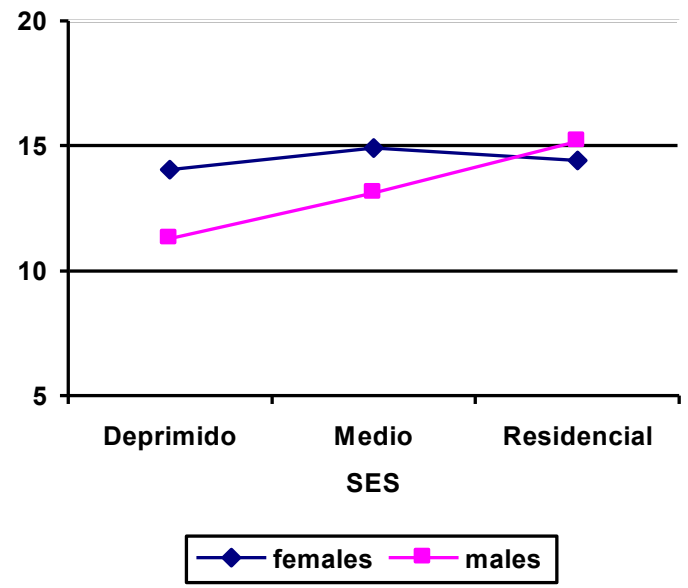

\section{Discusión}

\section{Efectos del Género}

En este trabajo se ha obtenido un efecto principal del género, según el cual los chicos muestran altas puntuaciones en Impulsividad autoevaluada. Las diferencias en función del género no varían según el contexto social: deprimido, medio y residencial. Este resultado aparece en otros estudios que evalúan también el efecto del género y el contexto social sobre conductas impulsivas, distinguiendo entre contexto rural y urbano (Hope y Bierman, 1998).

El término Impulsividad se refiere a la conducta de actuar y decir cosas antes de ser conscientes del riesgo que implican (Eysenck, Easting y Person, 1984). Impulsividad es un criterio clave para el diagnóstico en el Diagnostic and Statistical Manual of Mental Disorders (American Psychiatry Association, 1987). Además existe una amplia literatura que documenta 
las relaciones entre impulsividad, conducta agresiva, antisocial, o delincuente (Farrington, 1989; Luengo, Carrillo de la Peña, Otero, y Romero, 1994). Además, la impulsividad es parcialmente responsable de la puntuación dada a los varones, menor que a las chicas, por los profesores cuando evalúan la competencia social de sus alumnos (Dykeman, C., Daehlin, W., Doyle, S. y Flamer, H.S., 1996). En la investigación sobre la solución de problemas interpersonales, las respuestas automáticas e impulsivas están negativamente relacionadas con la competencia social. Al responder impulsivamente, los niños varones agresivos proporcionan pocas soluciones verbalmente asertivas, y más soluciones de escalamiento del conflicto, comparados con sus iguales no agresivos (Rabiner, Lenhart y Lochman, 1990). Pero esta relación no se encuentra en las chicas.

En nuestro estudio, en el que utilizamos autoevaluación, parece que pueden derivarse dos consecuencias de los resultados: a) la medida autoevaluada utilizada aquí, coincide con los resultados de profesores e iguales en otros estudios, al identificar a los varones como más impulsivos que las chicas; b) no aparece ninguna otra diferencia entre chicos y chicas en las variables utilizadas en este estudio. Quizás las diferencias son más sutiles y podrían aparecer en estudios más exhaustivos. Sin embargo debe señalarse que aparecen diferencias por género también en empatía pero en interacción con el efecto del contexto social.

\section{Efectos del contexto social}

El contexto social produce más diferencias que el género, algunas de las cuales no eran esperadas. El hecho de que la puntuación en Habilidades Sociales Apropiadas autoinformadas del grupo de contexto social deprimido sea superior a la del grupo de contexto residencial es sorprendente, pues otros estudios basados en evaluación de iguales indican que los adolescentes que viven en contextos sociales deprimidos es más probable que hayan aprendido patrones de interacción con padres e iguales más agresivos e inapropiados (Heimer, 1997) y niños que crecen en entornos económicamente deprimidos representan una población con elevado riesgo de desarrollar conductas agresivas y problemáticas (Farrington, 1991). En adición, cabe comentar que el grupo de contexto medio también puntúa más alto que el grupo de contexto residencial en esta variable.

Para explicar este resultado es quizás preciso atender a los elementos subjetivos de la evaluación de competencia social, los cuales se alojan en el dominio personal más que en las 
restricciones externas, tales como creencias en valores sociales, en criterios morales y sobre lo que está bien o mal (Hawkins, Catalano, Morrinson, O’Donnell y Day, 1992). Teniendo en cuenta el importante peso de estos elementos en la autoevaluación, sugerimos que es posible que los niños de contexto residencial hayan interiorizado reglas más exigentes por lo que se juzgan más severamente que los de los otros dos grupos, en relación a los criterios específicos de la competencia social. Es decir, este grupo podría ser más crítico hacia su conducta debido a que hubieran aprendido criterios más exigentes para definir la conducta social apropiada, propios de los contextos más acomodados.

Una explicación alternativa puede, basándose en recientes estudios, destacar que bajas puntuaciones en escalas de autoestima podrían reflejar una orientación más cauta, conservadora y de autoprotección mientras que altas puntuaciones podrían ser una manifestación de un estilo más arriesgado de engrandecimiento en la presentación del Yo (Baumeister, Smart y Boden, 1996; Colvin, Bloch y Funder, 1996; Raskin, Novacek y Hogan, 1991; Salmivalli, Kauliainen, Kaistaniemi y Lagerspetz, 1999 ) e incluso una manifestación de mecanismos de defensa (Schneider y Turkat, 1975). Cualquiera de estas tendencias dificultaría la evaluación de una saludable o genuina autoestima, y requeriría otro tipo de instrumentos diferentes a la escala MESSY utilizada en este estudio.

Un punto débil en la explicación anterior está en la alta puntuación en sobreconfianza del grupo de contexto residencial. Tal factor, a causa de contenidos como "me jacto de mi mismo," "pienso que se todo," o "actúo como si fuera mejor que otras gentes," parece referirse a un punto de vista narcisístico, de engrandecimiento del Yo, causado por un rechazo defensivo a creer nada negativo sobre si mismo, o por búsqueda constante de atención y de realce de si mismo. Los otros dos items en este factor de sobreconfianza: "permanezco con otros más tiempo del necesario" y “explico las cosas más tiempo de la cuenta” representan un punto de vista crítico hacia el Yo. Sin embargo, estudios culturales muestran que la gente en la cultura occidental, da importancia a los propios éxitos, explica mucho tiempo sus fracasos, intentando enaltecerse a si mismos (Miller, 1986; Whitley y Frieze, 1985) por lo que muestran a menudo sesgos favorables para su Yo (Harter, 1990). 
Sin embargo, independientemente de la duda de si la variable sobreconfianza tiene o no un carácter saludable, parece difícil de explicar porqué los alumnos de contexto residencial tienen puntuaciones más bajas que los otros dos grupos en Habilidades Sociales Apropiadas.

Ahora examinamos las conductas agresiva, pasiva, asertiva hacia adultos e iguales. El modelo de Michelson et al (1983), en el cual se basa la prueba aplicada en este estudio, indica que la respuesta asertiva supone un balance entre la imposición de nuestras necesidades a otros, lo que sería conducta agresiva, y en el otro extremo ceder y aguantar, lo que sería la respuesta pasiva. Hay una relación entre estas tres variables pues una mayor asertividad supone una agresividad o respuesta pasiva bajas. Puede deducirse que una respuesta asertiva es más socializada que una respuesta agresiva, por lo que los autores defienden la educación de asertividad como medio para prevenir violencia en niños y adolescentes. El modelo también asume que las relaciones con profesores y adultos ocurren en un diferente contexto que las que son con iguales.

En nuestros datos sólo las respuestas referidas a adultos son afectadas por la variable de contexto social. Sólo los alumnos de clase acomodada muestran relaciones de acuerdo a lo esperado según el modelo. Estos alumnos puntúan más alto en asertividad y más bajo en pasividad y agresividad en relaciones con adultos. Asertividad es una habilidad social que evita agresividad porque permite expresar necesidades sin interferir con los derechos de los demás, siguiendo las normas de cortesía o educación (Brown y Levinson, 1987). Teniendo en cuenta que lo que se considera conducta asertiva es definido por reglas sociales específicas para diferentes situaciones y contextos, puede pensarse que las normas de clase acomodada permitan y animen la conducta asertiva como más habilidosa en la expresión de sentimientos, deseos, opiniones o necesidades que la respuesta agresiva.

El grupo de contexto medio también muestra un patrón coherente de relaciones entre estas tres variables, diferente al del grupo residencial. Este se caracteriza por una baja puntuación en agresividad y asertividad con adultos y una alta puntuación en pasividad, sugiriendo que, en estos contextos quizás, la actitud pasiva puede ser vista como de respeto tradicional por profesores y padres, siendo más valorada. 
Es el grupo de contexto deprimido el que muestra los resultados más incoherentes, con una alta puntuación en agresividad, alta en asertividad y media en pasividad en las relaciones con adultos. Esto no parece ser una situación saludable. Además, una alta agresividad hacia los profesores y adultos puede ser una de las conductas inapropiadas peor consideradas en el entorno escolar. Incluso puede ser considerada peor que la agresividad hacia iguales. El hecho de que los alumnos de contexto deprimido puntúen más alto en esta medida que los otros dos grupos puede ser porque estos tres contextos pueden representar comunidades distintas, en cuanto a los contextos escolares que albergan.

Los efectos de contextos urbanos violentos y la densa congregación de alumnos de alto riesgo en las clases, son factores que pueden incrementar el riesgo de desarrollar problemas de conducta violenta y disruptiva en escuelas en contextos socioeconómicamente bajos (Hope y Bierman, 1998). Igualmente Furlong, Babiski, Poland, Muñoz y Boles, (1996) informan que psicólogos escolares encuentran más problemas de violencia en escuelas de áreas deprimidas (13.4\%) que en áreas residenciales (4.9\%) o de tipo medio (0\%). También en España existe la creencia fundada de que las escuelas de entornos deprimidos presentan más problemas de violencia que las de entornos medios o acomodados.

No podemos explicar porqué los alumnos de contexto deprimido presentan, al mismo tiempo altas puntuaciones en asertividad y agresividad y puntuación media en pasividad hacia profesores y adultos. Estos resultados parecen contradecir las relaciones esperadas según el modelo de Michelson et al. Quizás el balance entre poder/ sumisión establecido en estos contextos deprimidos sea diferente, y un alumno con problemas interpersonales con los adultos se comporte primero agresiva y asertivamente, y según se intensifique la respuesta del adulto el alumno se vaya controlando hasta llegar a mostrar conformidad y pasividad. Esto explicaría que, en una análisis sincrónico, las tres puntuaciones parezcan funcionar independientemente entre sí. En cualquier caso, en los contextos deprimidos, los adolescentes están expuestos a modelos agresivos y es probable que hayan sido reforzados en su conducta agresiva, según creencias normativas que apoyan el empleo de conducta agresiva para resolver problemas interpersonales, a diferencia de los otros dos contextos estudiados.

En el caso de las respuestas hacia iguales sólo la respuesta agresiva muestra resultados significativos. Debe tenerse en cuenta que el contexto de iguales es diferente al de los adultos, a la hora de analizar las medias de los diferentes contextos estudiados. El grupo de contexto 
residencial puntúa más alto que los otros dos grupos en agresividad hacia iguales. Este grupo, que tiene la más baja puntuación en agresividad hacia adultos, cambia completamente cuando se evalúan las relaciones con iguales. También el grupo medio y el deprimido cambian sus medias desde un tipo de relaciones al otro. Pero mientras el grupo medio incrementa su puntuación en agresividad al referirse a las relaciones con iguales, el grupo deprimido la disminuye. Estos resultados nos conducen a suponer que los criterios para la solución de problemas interpersonales varían tanto según el tipo de relación implicada como según el tipo de contexto social de referencia.

\section{Interacción entre género y contexto social}

Tres variables registran el efecto de la interacción entre género y contexto social. Una de ellas es la Empatía autopercibida respecto de la cual está bien establecida la superior puntuación en chicas que en chicos (Switzer, Simmons, Dew, Regalski y Wang, 1995). En nuestros datos, las chicas muestran igual media que los chicos en el grupo de contexto medio, pero difieren, mostrando superior media en los otros dos grupos. Traustadottir (1991) supone que la superior imagen de la mujer, como más empática y prosocial que los varones puede ser explicada por los diferentes procesos de socialización de hombres y mujeres. Entre las responsabilidades primarias de las mujeres se sigue considerando las tareas familiares. Con el tiempo esas normas son interiorizadas, llegando a ser las mujeres más afectuosas y hábiles para cuidar a otros que los varones (Eagly, 1987; Eagly y Crowley, 1986).

Otra variable que acusa la interacción entre género y contexto social es Asertividad Inapropiada. Este resultado puede comentarse desde dos perspectivas. Por una parte, sólo en el grupo deprimido se diferencian las medias de los chicos y las chicas. Una explicación podría ser que en el contexto deprimido se mantiene una diferente consideración social para estas conductas en la chica y en el chico, mientras que en los otros dos contextos estas diferentes evaluaciones tienden a converger. En el caso de la tercera variable que acusa la interacción que es Celos/ Soledad, también se producen más diferencias entre chico y chica, en el contexto deprimido y ninguna en el residencial.

Otro aspecto a comentar es que las puntuaciones más alta tanto en chica como en chico las muestra el grupo de contexto residencial. Este factor contiene ítems referidos a las relaciones con iguales, que van desde respuestas agresivas tales como "amenazar a alguien o 
actuar como matón," o "me meto mucho en peleas," hasta respuestas que vulneran normas de cortesía o educación como "hablo demasiado fuerte," o "me burlo de otros." El grupo residencial (sin diferenciar el género) también puntuaba más alto que los otros en agresividad hacia iguales en el test de Michelson et al (1983). Todos estos resultados están, sin duda, relacionados, y tienen que ver con diferentes criterios para juzgar las conducta inhábil/ hábil en cada contexto, como ya se ha comentado antes. En el caso de la puntuación en Celos/ Soledad también es superior en el grupo residencial.

Este trabajo enfatiza las contradicciones e interacciones internas de la autoevaluación entre niños de 11-12 años, casi preadolescentes. El empleo de autoinformes tiene sin duda riesgos de sesgos personales y está sujeto a errores cognitivos, defensivos o de autoengrandecimiento del Yo. Estos sesgos pueden distorsionar las medias. Pero por otro lado es necesario para conocer el punto de vista preadolescentes, que tiene un gran valor cara al diagnóstico y tratamiento de problemas en las relaciones sociales. Cabe señalar que la perspectiva del género no arroja diferencias sin la interacción con el contexto social, el cual presenta mayor efecto sobre la autovaloración de competencia social, en nuestro estudio. Nos conduce a señalar la necesidad de evaluar las competencias personales, con todas las complejas relaciones que muestran con diferentes factores del microsistema subjetivo.

Este trabajo podría ser mejorado incluyendo medidas de autoconcepto y autoestima menos tradicionales, que ayudasen a clarificar la interpretación de las puntuaciones respecto a las perspectivas defensivas o de autoengrandecimiento del Yo, que no deben ser consideradas hábiles. Finalmente, al evaluar las características de los contextos sociales, puede resultar de ayuda investigar las normas y valores que rigen las relaciones sociales, para poder interpretar con mayor exactitud las diferencias encontradas entre sujetos pertenecientes a diferentes contextos. 


\section{REFERENCIAS}

American Psychiatry Association (1987). Diagnostic and statistical manual of mental disorders ( $3^{\text {rd }}$ ed., rev.). Washington, DC: Author.

Baumeister, R., Smart, L. y Boden, J. (1996). Relations of threatened egotism to violence and aggression: The dark side of high self-esteem. Psychological Review, 103, 5-33.

Bracken, B.A. y Crain, R.M. (1994). Children's and adolescents' interpersonal relations: Do race and gender define normalcy? Journal of Psychoeducational Assessment, 12, 14-32.

Brown, P. y Levinson, S. (1987). Politeness: some universals in language usage. Cambridge: Cambridge University Press.

Bryant, B.K. (1982). An index of empathy for children and adolescents. Child Development. 53, 413-425.

Cavell, T.A. (1990). Social adjustment, social performance and social skills: A tri-component model of social competence. Journal of Clinical Child Psychology, 19 (2), 111-122.

Cichetti, D. y Bukowski, W.M. (1995). Developmental processes in peer relations and psychopathology. Development and Psychopathology, 7, 587-589.

Colvin, C.R., Block, J. y Funder, D.C. (1995). Overly positive self-evaluations and personality: negative implications for mental health. Journal of Personality and Psychology, 68, 1152-1162.

Dykeman, C., Daehlin, Doyle y Snow, (1996). Psychological predictors of school-based violence: Implications for school counsellors. The School Counsellor, 44, 35-47.

Eagly, A. (1987). Sex differences in social behaviour: A social role interpretation. Hillsdale, NJ: Lawrence Earlbaum.

Eagly, A. y Crowley, M. (1986). Gender and helping behaviour: A metaanalytic review of the social psychological literature. Psychological Bulletin, 1, 283-308.

Eysenck, S.B., Easting, G. y Pearson, P.R. (1984). Age norms for impulsiveness, venturesomeness and empathy in children. Personality and Individual Differences, 5, 315321.

Farrington, D.P. (1991). Childhood aggression and adult violence: Early precursors and later life outcomes. En D.J. Pepler y K.H. Rubin (Eds.), The development and treatment of childhood aggression (pp. 5-29). Hillsdale, NJ: Erlbaum.

Furlong, M., Babinski, L., Poland, S., Muñoz, J. y Boles, S. (1996) Factors associated with school psychologists' perceptions of campus violence. Psychology in the Schools, 33, 28-37.

Guerra, N.G., Huesmann, L.R, Tolan, P.H., Van Acker, R. y Eron, L.D. (1995). Stressful events and individual beliefs as correlates of economic disadvantage and aggression among urban children. Journal of Consulting and Clinical Psychology, 63 (4), 518528.

Hawkins, J.D., Catalano, R.F. \& Associates (1992) Communities that care: Action for drug abuse prevention. San Francisco: Guilford Press.

Heimer, K. (1997). Socio-economic status, subcultural definitions, and violent delinquency. Social Forces, 75(3), 799-833.

Helsel, W.J. y Matson, J.L. (1984). The assessment of depression of children: The internal structure of the Children's Depression Inventory (CDI). Behavior Research and Therapy, 22, 289-298.

Hope, T.L. y Bierman, K.L. (1998). Patterns of home and school behaviour problems in rural and urban settings. Journal of School Psychology, 36 (1), 45-58. 
Jaffe, P., Wolfe, D., Wilson, S. y Zak, L. (1985). Critical issues in the assessment of children's adjustment to witnessing family violence. Canada's Mental Health, 33 (4), 15-19.

Kazdin, A.E., Matson, J.L. y Esveldt-Dawson, K. (1984). The relationship of role-play assessment of children's social skills to multiple measures of social competence. Behaviour Research and Therapy, 22, 129-140.

Koestner, R. y Aube, J. (1995). A multifactorial approach to he study of gender characteristics. Journal of Personality, 63 (3), 681-710.

Luengo, M.A., Carrillo de la Peña, M.T., Otero, J.M. y Romero, E. (1994). A short-term longitudinal study of impulsivity and antisocial behaviour. Journal of Personality and Social Psychology, 66, 542-548.

McConnell, R.S., y Odom, S.L. (1986). Sociometrics: Peer-referenced measures and the assessment of social competence. En P. S. Strain, M. J. Guralnick, y H. H. Walker (Eds.), Children's Social Behavior (pp. 215-284). New York: Academic Press.

Matson, J.L., Macklin, G.F. y Helsel, W.J. (1985). Psychometric properties of the Matson Evaluation of Social Skills with Youngsters (MESSY) with emotional problems and self concept in deaf children. Journal of Behavior Therapy and Experimental Psychiatry, 16, 117-123.

Matson, J.L., y Ollendick, T.H. (1988). Enhancing children's social skills. New York: Pergamon Press.

Matson, J.L.; Rotatori, A.F.; and Helsel, W.J. (1983). Development of a rating scale to measure social skills in children: the Matson evaluation of social skills with youngsters (MESSY). Behaviour Research Therapy, 21(49), 335-34O.

Méndez, F.X., Hidalgo, M.D. e Inglés, C.J. (2002). The Matson Evaluation of Social Skills with Youngsters: Psychometric properties of the Spanish translation in the adolescent population. European Journal of Psychological Assessment, 18(1), 30-42.

Michelson, L., S., P., Wood, R.P., y Kazdin, A.E. (1983). Social skills assessment and training with children. New York: Plenum Press.

Miller, J. (1986). Toward a new psychology of women ( $2^{\text {nd }}$ ed.). Boston: Beacon Press.

Monjas, M.I. (2002). La promoción de competencia social ¿moda pasajera o necesidad?. Actas de las Jornadas sobre habilidades sociales ( pp. 37-50). Valladolid: Universidad.

Parker, J.G., Rubin, K.H., Price, J.M. y DeRosier, M.E. (1995). Peer relationships, child development, and adjustment: a developmental psychopathology perspective. En D. Cicchetti y D.I. Cohen (Eds.), Developmental psychopathology. Vol. II (pp.96-161). New York: Wiley.

Prior, M., Smart, D., Sanson, A., y Oberklaid, F. (1993). Sex differences in psychological adjustment from infancy to 8 years. Journal of the American Academy of Child and Adolescent Psychiatry, 32 (2), 291-304.

Rabiner, D.L., Lenhart, L. y Lochman, J.E. (1990). Automatic versus reflective social problem solving in relation to children's sociometric status. Developmental Psychology, 26(6), 1010-1016.

Raskin, R., Novacek, J. y Hogan, R. (1991). Narcissistic self-esteem management. Journal of Personality and Social Psychology, 60, 911-918.

Roberts, L., Gerritz, B., y Kearney, K. (1990). Shaping your school's culture to improve student learning. Los Angeles, CA: California School Leadership Academy.

Salmivalli, C., Kaukiainen, A, Kaistaniemi, L. y Lagerspetz, K.M.J. (1999). Self-evaluated self-esteem, peer-evaluated self-esteem, and defensive egotism as predictors of adolescents' participation in bullying situations. Personality and Social Psychology Bulletin, 2 5(10), 1268-1278. 
Schneider, D., y Turkat, D. (1975). Self-presentation following success or failure: Defensive self-esteem models. Journal of Personality and Social Psychology, 43, 127-135.

Soriano, M. y Soriano, F.I. (1994). School violence among culturally diverse populations: Sociocultural and institutional considerations. School Psychology Review, 23(2), 216235.

Spence, S.H. y Liddle, b. (1990). Self-report measures of social competence for children: An evaluation of Social Skills for Youngsters and the List of Social Situation Problems. Behavioral Assessment, 12, 317-336.

Switzer, G.E., Simmons, R.G., Dew, M.A., Regalski, J.M. y Wang, Ch-H (1995). The effect of a school-based helper program on adolescent self-image, attitudes and behaviour. Journal of Early Adolescence, 15(4), 429-455.

Traustadottir, R. (1991). Mothers who care: Gender, disability and family life. Journal of Family Issues. 12(2), 211 -228.

Trianes, M.V. (1996). Educación y competencia social: un programa en el aula. Málaga: Aljibe.

Trianes, M.V., Blanca, M.J., Muñoz, A., García, B., Cardelle-Elawar, M. e Infante, L. (2002) Relaciones entre evaluadores de la competencia social en preadolescentes: Profesores, iguales y autoinformes. Anales de Psicología, 18(2), 197-214.

Trianes, M.V., Muñoz, A., Blanca,M.J., Sánchez, A. y García, B., (1999, Septiembre). ¿Cómo se relacionan medidas situacionales de asertividad escolar con otras variables de competencia social?. III Congreso Internacional de Psicología y Educación, Santiago de Compostela: Facultad de Educación U. Complutense.

Trianes, M.V. y Fernández-Figarés, C. (2001). Aprender a ser persona y a convivir: Un programa para secundaria. Bilbao: Descleé de Brower.

Verhalst, F.C., Akkerhuis, G.W. y Althaus, M. (1985). Mental health in Dutch children: A cross-cultural comparison. Acta Psychiatrica Scandinavica, 72. suppl: 108 pp.

Whitley, B.E. Jr., y Frieze, I.H. (1985). Children's causal attributions for success and failure in achievement settings: A meta-analysis. Journal of Educational Psychology, 77, 608616.

Note:

This paper was presented at the Arizona Educational Research Organisation, 1997. Este trabajo ha sido financiado por el Ministerio de Educación y Ciencia (DGICYT: PB93-0987). 
Esta página está en blanco intencionadamente. 